\title{
Detection of Single-Copy Chromosome 17q Gain in Human Neuroblastomas Using Real-Time Quantitative Polymerase Chain Reaction
}

\author{
Michael Morowitz, M.D., Suzanne Shusterman, M.D., Yael Mosse, M.D., George Hii, B.S., \\ Cynthia L. Winter, B.A., Deepa Khazi, M.S., Qun Wang, M.D., Ph.D., Rebecca King, B.S., \\ John M. Maris, M.D. \\ Division of Oncology, Children's Hospital of Philadelphia (MM, SS, GH, RK, CLW, DK, QW, YM, JMM); \\ and Departments of Pediatrics (SS, YM, JMM) and of Surgery (MM), University of Pennsylvania School of \\ Medicine, Philadelphia, Pennsylvania
}

Regional genomic alterations resulting from singlecopy allelic loss or gain have been well characterized in many human cancers and are often of prognostic relevance. Unbalanced gain of $17 \mathrm{q}$ material is common in malignant human neuroblastomas and typically results from unbalanced translocations. Unbalanced $17 q$ gain may be an independent predictor of disease outcome, but technical difficulties with quantifying such gain using fluorescent in situ hybridization gives this method limited clinical applicability. We now describe a duplex genomic DNAbased quantitative polymerase chain reaction assay to determine the presence or absence of unbalanced gain of chromosome 17q in primary neuroblastoma specimens. The technique was first refined and validated in a panel of nine human neuroblastomaderived cell lines by direct comparison with dualcolor fluorescent in situ hybridization. Prospective blinded comparison of quantitative polymerase chain reaction and fluorescence in situ hybridization in $\mathbf{4 0}$ human neuroblastoma primary tumor samples showed a sensitivity of $96 \%$ and $100 \%$ specificity for detecting unbalanced $17 q$ gain when a relative $17 q$ copy number ratio of 1.3 was used to define unbalanced gain. Tumors with ratios $>1.3$ were highly associated with malignant tumor phenotypic features such as metastatic disease $(P<$ $.0001)$ and tumor $M Y C N$ amplification $(P=.008)$. These data suggest that quantitative polymerase chain reaction determination of $17 q$ status is feasi-

\footnotetext{
Copyright () 2003 by The United States and Canadian Academy of Pathology, Inc.

VOL. 16, NO. 12, P. 1248, 2003 Printed in the U.S.A.

Date of acceptance: August 15, 2003.

Authors Morowitz and Shusterman contributed equally to this work. Address reprint requests to: John M. Maris, M.D., Division of Oncology, Children's Hospital of Philadelphia, Abramson Research Center, Room 902A, 34th and Civic Center Blvd., Philadelphia, PA 19104-4318; fax: 215-590-3770; e-mail: maris@email.chop.edu.

DOI: 10.1097/01.MP.0000097364.64566.81
}

ble and highly specific in primary tumor samples. Sensitivity may be limited because of the inherent complexity of both the chromosomal rearrangements and heterogeneity of some tumor samples. Taken together, quantitative polymerase chain reaction can be used as a high-throughput screening tool for $17 q$ aberrations, but a subset of samples may also require fluorescence in situ hybridization analysis in an attempt to conclusively determine $17 q$ allelic status.

KEY WORDS: Chromosome 17, Fluorescence in situ hybridization, Genetics, MYCN, Neuroblastoma, Quantitative PCR, Unbalanced gain.

Mod Pathol 2003;16(12):1248-1256

Neuroblastoma is the most common malignant disease of infancy and the third most common pediatric cancer overall (1). It is a markedly heterogeneous disease, characterized by a wide diversity in natural history and treatment outcomes. Although patient age at diagnosis and stage of the tumor have been demonstrated to be important variables for predicting survival, it is clear that cellular and molecular features of individual primary tumors convey important additional prognostic information (2). For example, the observation that MYCN amplification is associated with advanced stage of disease and rapid tumor progression $(3,4)$ has resulted in the current standard of care of testing all diagnostic neuroblastoma samples for this genomic aberration to guide treatment planning. The Children's Oncology Group, as well as most international pediatric oncology cooperative groups, considers age at diagnosis, disease stage, Shimada histopathologic classification, DNA index, and MYCN amplification status in assigning patients a risk category for subsequent treatment planning $(1,5,6)$. However, risk classification using these five prognostic variables 
remains imprecise, and current research is focused on identifying additional tumor-specific prognostic variables that will improve the ability to properly stratify patients for treatment planning.

Unbalanced gain of $17 \mathrm{q}$ genomic material is a common somatically acquired abnormality in primary neuroblastomas (7-9). It has been demonstrated that these aberrations are frequently caused by unbalanced translocations that concomitantly result in loss of distal $1 \mathrm{p}, 11 \mathrm{q}$, or other regions of recurrent allelic loss $(7,10-14)$. Unbalanced $17 q$ gain is present at diagnosis in the more aggressive subset of neuroblastomas, suggesting that this is a somatically acquired event that contributes to a more malignant phenotype. Furthermore, the review by Bown and colleagues of 313 cases from European centers showed that unbalanced 17q gain detected by FISH was an independent marker for disease outcome (9). These data strongly suggest that risk stratification can be refined by reliable assessment of $17 \mathrm{q}$ allelic status in primary neuroblastoma specimens.

Dual-colored interphase FISH on tumor touch preparations appears to be a relatively sensitive and specific method for determining $17 \mathrm{q}$ allelic status, but this has not been tested formally. Routine FISH detection of $17 \mathrm{q}$ anomalies is difficult in the cooperative group setting because of the large volume of samples requiring analysis, low throughput, high cost, inadequate diagnostic material in many cases relative to what is required for high-quality touch preparations, and intratumoral heterogeneity, often making interpretation difficult. The Children's Oncology Group is interested in testing the independent prognostic impact of $17 q$ allelic status, especially because the previous report did not include Shimada histopathology as a covariate (9), and histopathologic classification is one of the cornerstones of the Children's Oncology Group risk stratification system (1). We therefore sought to develop a relatively simple and high-throughput technique using real-time quantitative PCR (Q-PCR), and thus requiring nanogram quantities of input DNA, to screen for unbalanced $17 \mathrm{q}$ gain in neuroblastoma specimens.

\section{MATERIALS AND METHODS}

\section{Samples and DNA Isolation}

Nine human neuroblastoma-derived cell lines were selected from the Children's Hospital of Philadelphia neuroblastoma cell line repository. Primary tissue samples from 40 diagnostic neuroblastoma specimens were randomly selected from the Children's Hospital of Philadelphia and Children's Oncology Group Neuroblastoma Tumor Banks. Each primary tumor sample was immediately snap- frozen in liquid nitrogen on receipt in the pathology lab, according to Children's Oncology Group guidelines. Clinical and biological variables including age at diagnosis, disease stage by the International Neuroblastoma Staging System (15), DNA index (6), and Shimada pathology (5) were available for all cases, and MYCN amplification status was available by FISH (16) in 38/40 cases. Tumor touch preparations were made from each primary tumor and fixed in 3:1 methanol:acetic acid. DNA was extracted by anion exchange chromotography (Qiagen, Valencia, CA). The Children's Hospital of Philadelphia Institutional Review Board approved this research.

\section{Real-Time Quantitative PCR}

Q-PCR amplimers were designed to be complementary to genomic regions that showed gain (17q) or loss (17p) in all previously reported cases of unbalanced $17 q$ gain $(9,11,17-20)$. Oligonucleotide primers and probes were designed with Primer Express v1.5 (Applied Biosystems, Foster City, CA) and were complimentary to the intron 1 genomic sequence of TP53 and the exon1-intron1 boundary within the genomic sequence of $M P O$ (Table 1). The probes were differentially labeled with fluorophores VIC and FAM to allow for duplex PCR reactions, and each probe was dual labeled with TAMRA to serve as a quencher dye.

Duplex Q-PCR was performed using an ABI PRISM 7700 Sequence Detector. Initial amplification mixtures $(25 \mu \mathrm{L})$ contained $10 \mathrm{ng}$ of genomic DNA, $1 \times$ TaqMan Universal PCR Master Mix (Applied Biosystems), $360 \mathrm{~nm}$ of each primer, and 100 nM of each probe. Final oligonucleotide concentrations were determined by optimization experiments in which primer concentrations were varied between $270 \mathrm{~nm}$ and $900 \mathrm{~nm}$ and probe concentrations were varied between $20 \mathrm{~nm}$ and $200 \mathrm{~nm}$ to identify combinations yielding optimal signal-tonoise and amplification efficiency. Cycling conditions were 2 minutes at $50^{\circ} \mathrm{C}$ and an initial denaturation step at $95^{\circ} \mathrm{C}$ for 10 minutes, followed by 40 cycles of $95^{\circ} \mathrm{C}$ for 15 seconds and $60^{\circ} \mathrm{C}$ for 60 seconds.

TABLE 1. Q-PCR Oligonucleotide Sequences and
Fluorescence Dyes




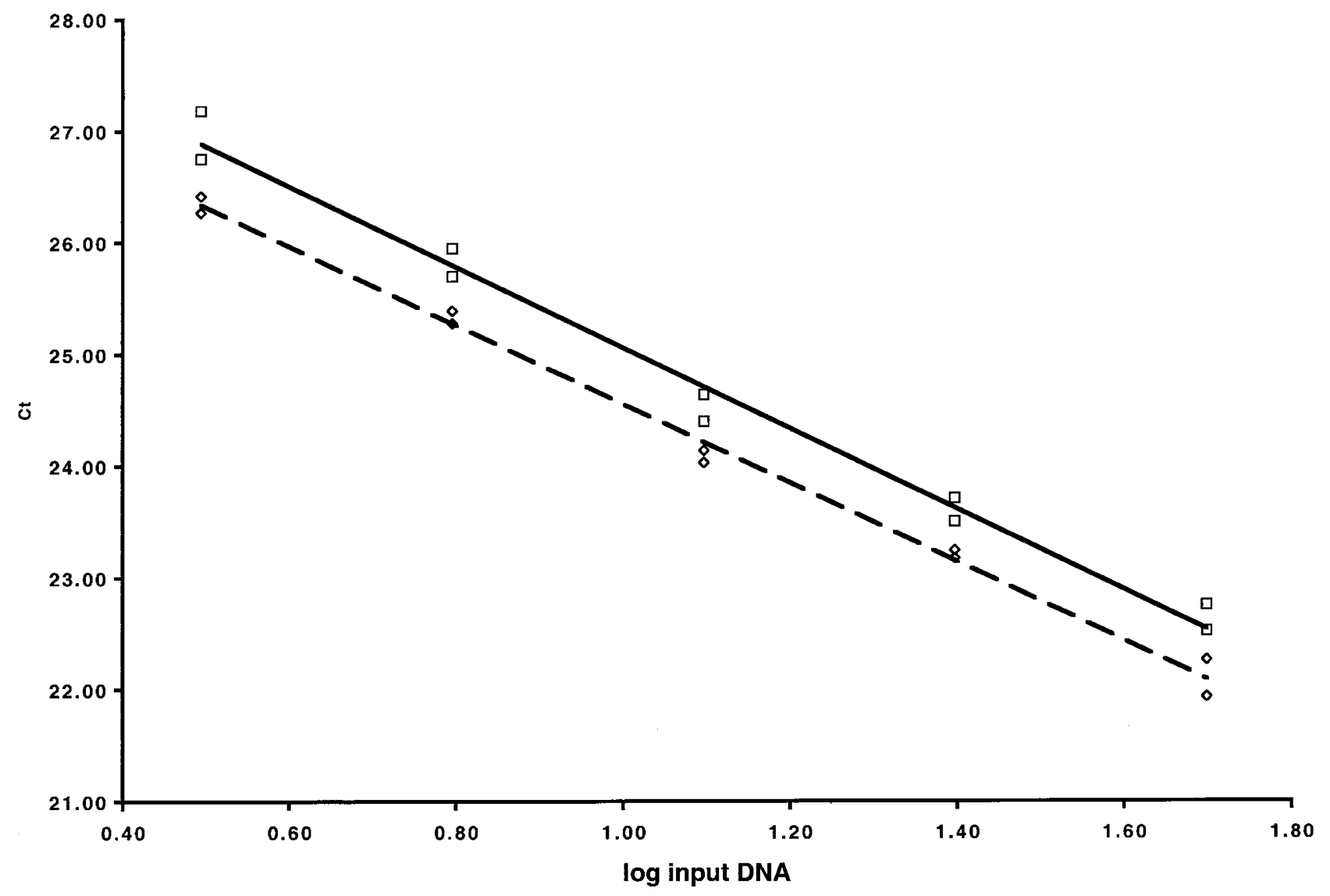

FIGURE 1. Quantitative PCR standard curves constructed with normal human genomic DNA. The amount of template input DNA is plotted against $\mathrm{C}_{\mathrm{T}}$ value for both TP53 (dashed) and MPO (solid). All reactions were performed in duplex with both sets of primers and probes. At each serial dilution, the difference between the TP53 and MPO $\mathrm{C}_{\mathrm{T}}$ values remains constant, indicating that the efficiencies of the reference amplification (TP53) and the target amplification (MPO) are equivalent. This relationship between the primer-probe sets validates the use of the comparative $\mathrm{C}_{\mathrm{T}}$ method for determining the presence of $17 q$ gain.

Initial Q-PCR experiments were performed using both the standard curve method and comparative threshold cycle $\left(\Delta \Delta \mathrm{C}_{\mathrm{T}}\right)$ method (21). Standard curves were constructed using serial dilutions of normal human genomic DNA from $50 \mathrm{ng}$ to $3.125 \mathrm{ng}$, and relative $M P O$ and TP53 copy numbers were extrapolated from the normal DNA standard curve using the $C_{T}$ value of an individual test sample. Relative $17 \mathrm{q}$ copy number by the comparative $\mathrm{C}_{\mathrm{T}}$ method was determined in both test and normal samples according to the following equation: $2^{-\Delta \Delta \mathrm{Ct}}=(1+\mathrm{E})^{-\Delta \mathrm{Ct} \text { sample/ }}$ $(1+E)^{-\Delta C t}$ calibrator, where the efficiency (E) of target and reference gene amplifications are equivalent (see validation below), $\Delta \mathrm{C}_{\text {Tsample }}$ is the difference in $\mathrm{C}_{\mathrm{T}}$ between the target gene and the reference gene in the test sample, and $\Delta \mathrm{C}_{\text {Tcalibrator }}$ is the difference in threshold cycle value between the target gene and the reference gene in the normal DNA sample.

\section{Dual-Color Interphase FISH}

FISH probes were designed to overlap the Q-PCR amplimer genomic regions. DNA extracted from human bacterial artificial chromosomes clones
RP11-89D11, mapping to the TP53 locus at 17p13.1, and RP11-506 21 , mapping to the $M P O$ locus at 17q23.1, were identified (Children's Hospital of Oakland Research Institute, Oakland, CA), and DNA from each clone was labeled with the Vysis Nick Translation Kit (Vysis, Inc., Downers Grove, IL). The TP53 and MPO probes were labeled with Spectrum Red and Spectrum Green dUTP, respectively. Dual-color interphase FISH was performed according to the manufacturer, with minor modifications. Briefly, slides were subject to denaturation at $73^{\circ} \mathrm{C}$ in a solution of $70 \%$ formamide in $2 \times$ standard saline citrate and then dehydrated in ethanol. Bacterial artificial chromosomes probes were precipitated in the presence of COT-1 DNA and then denatured for 5 minutes at $73^{\circ} \mathrm{C}$ in LSI/WCP Hybridization Buffer (Vysis, Inc., Downers Grove, IL). Ten microliters of each probe was applied concomitantly to tumor touch preparation slides and hybridized in a moist chamber at $37^{\circ} \mathrm{C}$ overnight. Slides were washed with $0.3 \% \mathrm{NP}-40 / 0.4 \times$ standard saline citrate at $73^{\circ} \mathrm{C}$, and then with $0.1 \% \mathrm{NP}-40 / 2 \times$ 
standard saline citrate at room temperature, and finally they were counterstained with DAPI II.

Fluorescence signals were visualized with a Zeiss Axiophot microscope (Jena, Germany), photographed with a computer-driven Photometrics Sensys color camera, and representative images were analyzed using the Powergene system. Hybridization signals from a minimum of 75 nuclei were counted for each sample. Samples were considered to have unbalanced gain of $17 \mathrm{q}$ ma- terial if $>50 \%$ of nuclei demonstrated one or more excess $17 \mathrm{q}$ signals relative to $17 \mathrm{p}$ signals, whereas $\leq 50 \%$ of nuclei with this pattern was considered no gain.

\section{Statistical Analyses}

Clinical and biological features were compared using the exact conditional $\chi^{2}$ test.

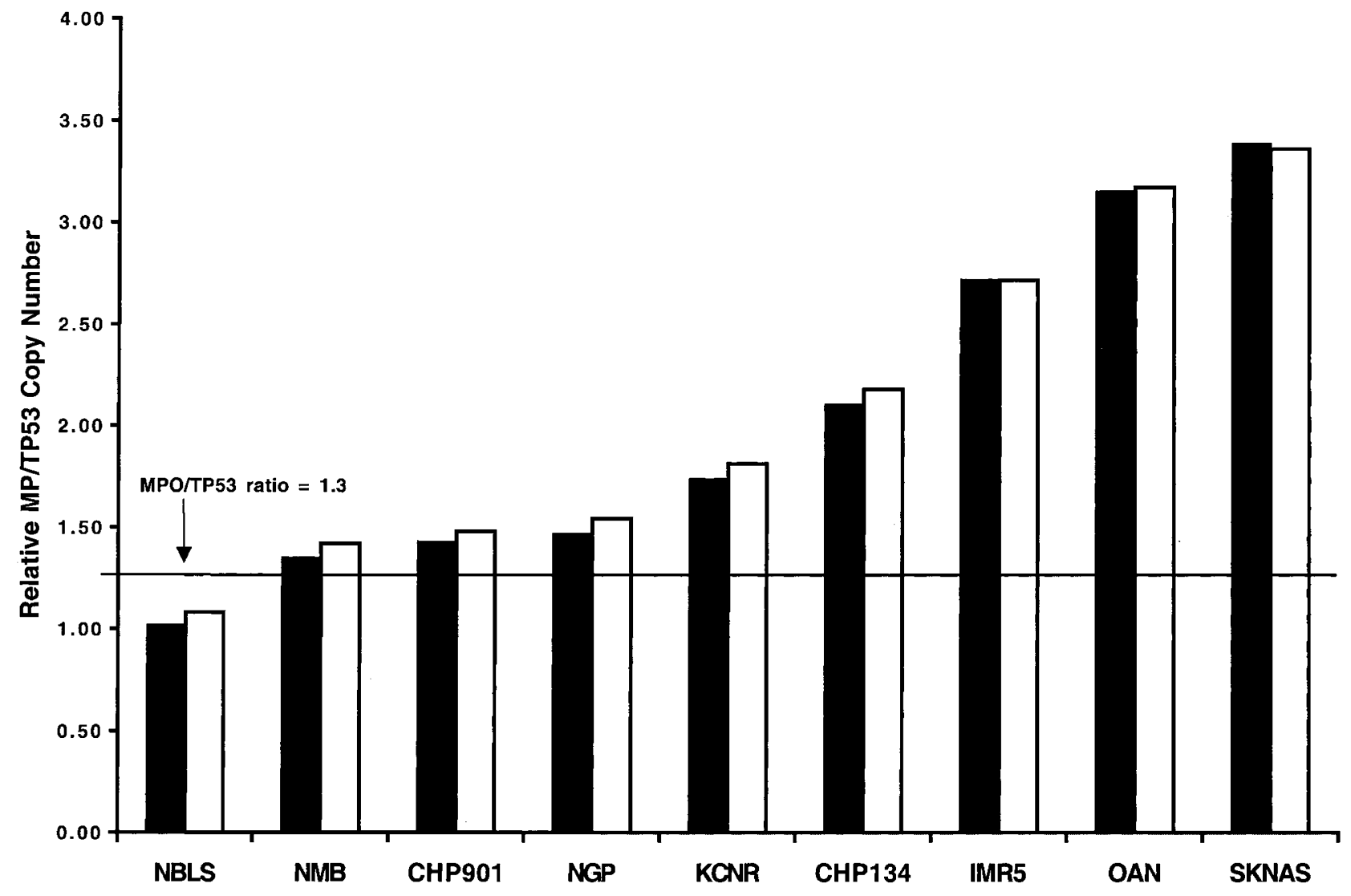

FIGURE 2. Comparison of standard curve and comparative $C_{T}$ methods for detecting $17 \mathrm{q}$ status in human neuroblastoma-derived cell lines. For each cell line tested, the ratio of MPO copy number to TP53 copy number is shown using both the comparative $\mathrm{C}_{\mathrm{T}}$ method (solid) and the standard curve method (open). All cell lines with unbalanced 17q gain by FISH showed relative 17q copy number ratios by Q-PCR of $>1.3$.

TABLE 2. Comparison of FISH and Q-PCR Results in Human Neuroblastoma-Derived Immortalized Cell Lines

\begin{tabular}{llccc}
\hline & \multicolumn{2}{c}{ FISH } & \multicolumn{2}{c}{ Real-Time PCR } \\
\cline { 2 - 3 } Cell Line & 17q Status & Pattern & $\begin{array}{c}\text { Std Curve } \\
\text { Result }\end{array}$ & 1.08 \\
Result
\end{tabular}

FISH results are reported by indicating the presence or absence of unbalanced 17q gain. Also shown is the predominant pattern of TP53 (17p) and $M P O$ (17q) fluorescence signals in the interphase nuclei. Real-time PCR results are reported as a ratio of MPO gene copy number to TP53 gene copy number, for both the standard curve and comparative Ct methods. 


\section{RESULTS}

\section{Optimization and Validation of Q-PCR in} Neuroblastoma Cell Lines

The optimal reaction concentrations determined in the serial dilution experiments were identical for both genes at $360 \mathrm{~nm}$ for each primer and $100 \mathrm{~nm}$ for each probe (data not shown). Duplex Q-PCR was then performed on serial dilutions of normal genomic DNA. PCR reaction kinetics were similarly efficient for each gene $(R \geq 0.99)$, suggesting that a comparative $\mathrm{C}_{\mathrm{T}}\left(\Delta \Delta \mathrm{C}_{\mathrm{T}}\right)$ method was an acceptable strategy for relative quantification of $17 \mathrm{q}$ copy number (Fig. 1) (21). Both the standard curve and the $\Delta \Delta \mathrm{C}_{\mathrm{T}}$ methods were used to determine $17 \mathrm{q}$ status in nine neuroblastoma cell lines. Results using both methods were essentially identical (Fig. 2; Table 2). The $\Delta \Delta \mathrm{C}_{\mathrm{T}}$ method was therefore used for further protocol development because it allows for higher sample throughput by obviating the need for serial dilutions in each experiment.

We next compared Q-PCR results with copy number determination by dual color interphase FISH. In our sample set of nine cell lines, NBLS was the only example of a normal $17 \mathrm{p}-17 \mathrm{q}$ allelic ratio by FISH (Fig. 2; Table 2), and this is not surprising because of the high correlation between $17 \mathrm{q}$ gain and the high-risk phenotype that is required to establish cell lines. This was the only sample that showed a relative $17 \mathrm{q}$ copy number determination of $<1.3$. The remaining samples showed a variety of copy number patterns by FISH with either a one- or two-copy difference between $17 \mathrm{q}$ and $17 \mathrm{p}$. Relative $17 \mathrm{q}$ copy numbers ranged from $1.35-3.39$, and these values in general correlated with the degree of discrepancy in $17 p$ and $17 q$ allelic copy number by FISH (Table 2).

\section{Prospective Evaluation of Q-PCR in} Neuroblastoma Primary Tumors

Q-PCR and dual-color interphase FISH were performed on 40 primary human neuroblastoma samples by individual investigators who were blinded to the results for the alternate technique. All samples were classified as unbalanced $17 \mathrm{q}$ gain present or absent by dual-colored FISH and Q-PCR (Fig. 3; Table 3). All samples could be categorized by FISH, although many samples had complex patterns of $17 q$ rearrangements with multiple distinct fluorescence signal patterns. Table 3 shows the predominant pattern observed. Q-PCR results ranged from 0.69-2.84 and correlated with the relative 17q copy number determination by FISH (Fig. 4). We explored different relative $17 \mathrm{q}$ copy number values for the binary determination of presence or absence of
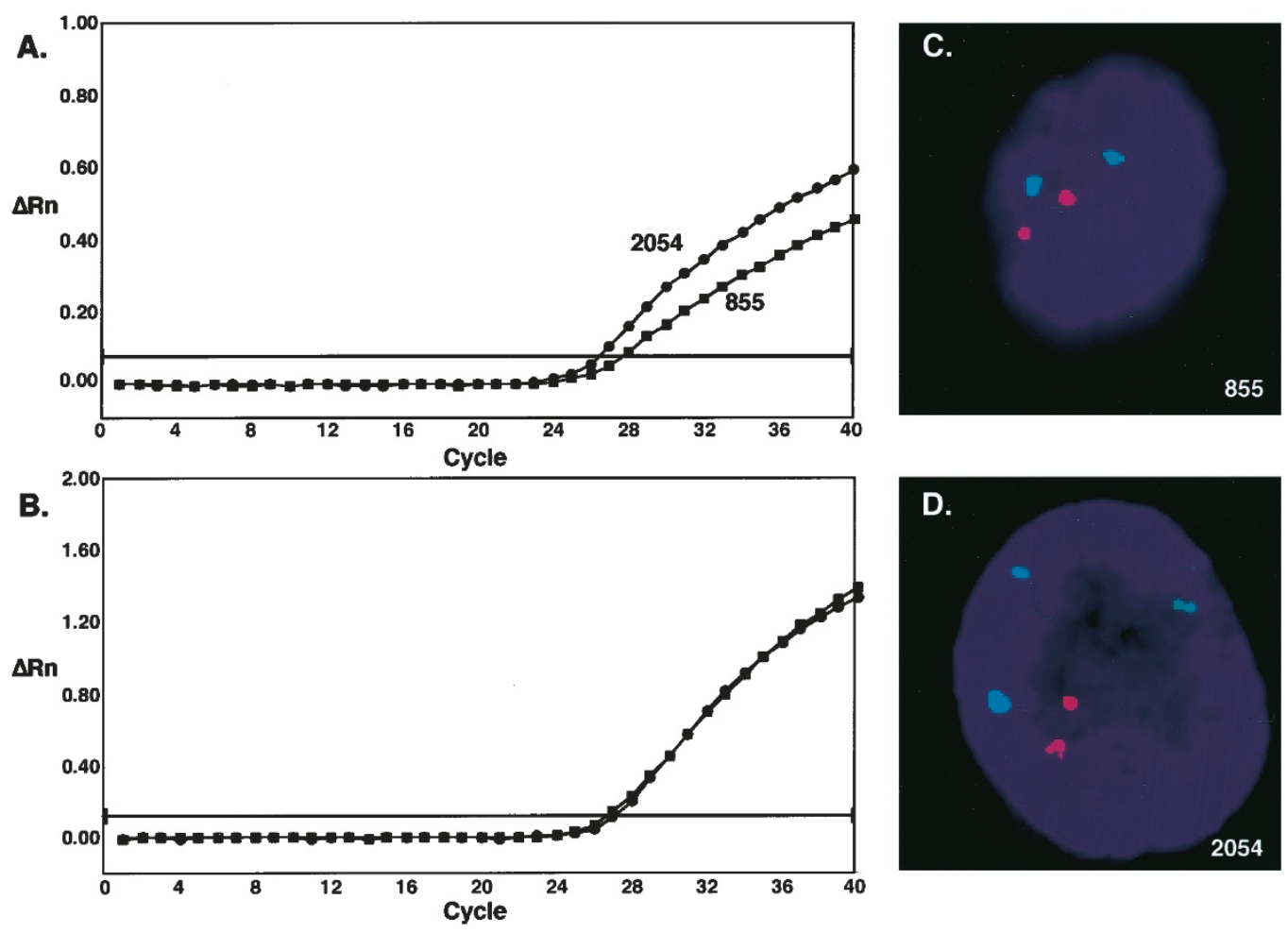

FIGURE 3. Correlation of Q-PCR and FISH in two primary tumors. Amplification plots of MPO (A) and TP53 (B) fluorescent signal versus cycle number for primary neuroblastomas 855 and 2054. The amplification plots are essentially identical for TP53, but tumor 2054 has a lower $\mathrm{C}_{\mathrm{T}}$ value for MPO. Using the $\Delta \Delta$ CT method, these results correspond to a $17 \mathrm{q}-17 \mathrm{p}$ ratio of 1.9 for tumor 2054 and of 1.0 for tumor 855 . These data are confirmed by dual-colored interphase FISH using probes to MPO (green) and TP53 (red). Shown are representative single nuclei showing no gain of 17q material for 855 (C) and unbalanced 17q gain for 2054 (D). 
unbalanced $17 q$ gain (Table 4 ). Using a relative $17 q$ copy number of 1.4 as a cut point showed $100 \%$ specificity (no false positives), but 6 false negatives (74\% sensitivity). Relaxing the stringency to 1.3 again showed $100 \%$ specificity with only 1 false negative (96\% sensitivity). Specificity decreased to $76 \%$ when the relative $17 q$ copy number cut point was further relaxed to 1.2 , but the sensitivity was $100 \%$.

Primary neuroblastomas with relative $17 \mathrm{q}$ copy number values of $>1.3$ were significantly more likely to be from patients with metastatic disease (Table 3; $P<.0001$ ). Concomitant MYCN amplification occurred exclusively in this group of tumors with high relative $17 \mathrm{q}$ copy number values $(P=$ .008). These tumors also tended to be from patients older than $1 \mathrm{y}$ of age $(P=.07)$.

\section{DISCUSSION}

Neuroblastoma is a clinically heterogeneous disease, and a large number of patients have an outstanding prognosis that can be predicted based on analysis of clinical and tumor biological variables. However, the majority of neuroblastoma cases show hematogenous metastases at diagnosis that is often refractory to treatment (1). Understanding the molecular features that discriminate the unfavorable from the favorable subsets of neuroblastoma has been a focus of intense research in recent years (reviewed in 2). To date, this research has identified a relatively large panel of somatically acquired genomic alterations that correlate with tumor natural history, including response to treatment. Neuroblastoma has served as a paradigm for the clinical

TABLE 3. Comparison of FISH and Q-PCR Results in Human Neuroblastoma-Primary Tumor Samples

\begin{tabular}{|c|c|c|c|c|c|c|}
\hline \multirow{2}{*}{ Sample } & \multirow{2}{*}{$\begin{array}{l}\text { Age at } \\
\text { Diagnosis } \\
\text { (days) }\end{array}$} & \multirow{2}{*}{ Stage } & \multirow{2}{*}{$\mathrm{MYCN}^{1}$} & \multicolumn{2}{|r|}{ FISH } & \multirow{2}{*}{$\frac{\text { Q-PCR }}{\Delta \Delta \text { CT Ratio }}$} \\
\hline & & & & $17 q$ Status $^{2}$ & Predominant Pattern (\%) & \\
\hline 305 & 736 & $2 b$ & Not Amp & No UG & $4 p 4 q(30 \%)$ & 0.69 \\
\hline 337 & 93 & 1 & Not Amp & No UG & $2 \mathrm{p} 2 \mathrm{q}(13.6 \%)$ & 0.90 \\
\hline 401 & 1846 & 3 & Not Amp & No UG & $4 q 4 p(56 \%)$ & 0.95 \\
\hline 249 & 173 & 3 & Not Amp & No UG & $2 p 2 q(46 \%)$ & 0.97 \\
\hline 314 & 218 & $2 b$ & Not Amp & No UG & $2 \mathrm{p} 2 \mathrm{q}(13.4 \%)$ & 0.97 \\
\hline 343 & 38 & 1 & Not Amp & No UG & 3p3q (19.3\%) & 1.00 \\
\hline 855 & 1747 & $2 b$ & Not Amp & No UG & $2 p 2 q(92 \%)$ & 1.00 \\
\hline 344 & 2980 & 3 & Not Amp & No UG & 3p3q (36\%) & 1.04 \\
\hline 3 & 417 & 3 & Not Amp & No UG & $2 p 2 q(91 \%)$ & 1.07 \\
\hline 756 & 5 & $4 \mathrm{~s}$ & Not Amp & No UG & $3 p 3 q(48 \%)$ & 1.07 \\
\hline 329 & 70 & 4 & Not Amp & No UG & $4 \mathrm{p} 4 \mathrm{q}(28.7 \%)$ & 1.09 \\
\hline 327 & 6 & $2 b$ & Not Amp & No UG & $4 \mathrm{p} 4 \mathrm{q}(8.9 \%)$ & 1.11 \\
\hline 1995 & 709 & $2 b$ & Not Amp & No UG & $4 p 4 q(73 \%)$ & 1.17 \\
\hline 796 & 377 & 3 & Not Known & UG & 2p3q (67\%) & 1.23 \\
\hline 351 & 1852 & 4 & Not Amp & No UG & $4 p 4 q(16.7 \%)$ & 1.23 \\
\hline 308 & 69 & 1 & Not Amp & No UG & 6p6q (19.3\%) & 1.25 \\
\hline 304 & 421 & $2 b$ & Not Amp & No UG & $4 p 4 q(20.8 \%)$ & 1.27 \\
\hline 2087 & 721 & 1 & Not Amp & No UG & $2 \mathrm{p} 2 \mathrm{q}(53 \%)$ & 1.28 \\
\hline 5 & 790 & 4 & Amp & UG & $3 p 4 q(79 \%)$ & 1.32 \\
\hline 320 & 514 & 4 & Amp & UG & 3p3q (19.8\%) & 1.33 \\
\hline 393 & 280 & 4 & Amp & UG & $2 p 4 q(25 \%)$ & 1.36 \\
\hline 334 & 907 & 4 & Not Amp & UG & $2 p 3 q(26.7 \%)$ & 1.38 \\
\hline 312 & 2269 & 4 & Not Amp & UG & $6 p 7 q(11.4 \%)$ & 1.39 \\
\hline 923 & 276 & 4 & Not Amp & UG & 2p3q (72\%) & 1.68 \\
\hline 1652 & 330 & 4 & Not Amp & UG & $2 p 3 q(70 \%)$ & 1.70 \\
\hline 340 & 916 & 4 & Not Amp & UG & $2 \mathrm{p} 4 \mathrm{q}(34.7 \%)$ & 1.75 \\
\hline 357 & 100 & $4 \mathrm{~s}$ & Not Amp & UG & $3 p 3 q(12.9 \%)^{3}$ & 1.76 \\
\hline 1673 & 485 & 4 & Not Amp & UG & $2 p 4 q(49 \%)$ & 1.81 \\
\hline 2054 & 1131 & 4 & Amp & UG & 2p3q (67\%) & 1.90 \\
\hline 306 & 659 & 4 & Not Amp & UG & $2 p 3 q(20.2 \%)$ & 1.92 \\
\hline 1554 & 1525 & 4 & Not Amp & UG & 2p5q (56\%) & 1.94 \\
\hline 1848 & 1555 & 4 & Not Amp & UG & $2 p 4 q(40 \%)$ & 1.96 \\
\hline 333 & 1189 & 4 & Not Amp & UG & $3 p 7 q(8.7 \%)$ & 2.06 \\
\hline 319 & 594 & 4 & Not Amp & UG & $2 \mathrm{p} 4 \mathrm{q}(58.5 \%)$ & 2.18 \\
\hline 1994 & 1422 & 4 & Amp & UG & $2 p 4 q(66 \%)$ & 2.49 \\
\hline 341 & 1002 & 4 & Not Amp & UG & 2p5q (50\%) & 2.51 \\
\hline 1749 & 841 & 4 & Not Known & UG & 3p6q (58\%) & 2.63 \\
\hline 317 & 895 & 4 & Amp & UG & $1 p 3 q(37.5 \%)$ & 2.74 \\
\hline 140 & 935 & 4 & Amp & UG & lp3q (72\%) & 2.78 \\
\hline 347 & 1070 & 4 & Not Amp & UG & $2 p 5 q(22.2 \%)$ & 2.84 \\
\hline
\end{tabular}

FISH results include the presence or absence of unbalanced gain of chromosome 17q material as well as the dominant hybridization. These are compared with the ratio of MPO (17q) gene dosage to TP53 (17p) gene dosage, as measured by Q-PCR.

${ }^{1}$ Amp = amplified; not amp = not amplified.

${ }^{2} \mathrm{UG}=$ unbalanced gain of chromosome $17 \mathrm{q}$; no $\mathrm{UG}=$ no gain or balanced gain of both $17 \mathrm{p}$ and $17 \mathrm{q}$.

${ }^{3}$ Sample 357 contained a heterogeneous sample of nuclei with a highly variable pattern of 17p and 17q FISH signals. Although 59\% of nuclei showed unbalanced gain of $17 \mathrm{q}$ material, the single most observed pattern of signals was 3p3q. 


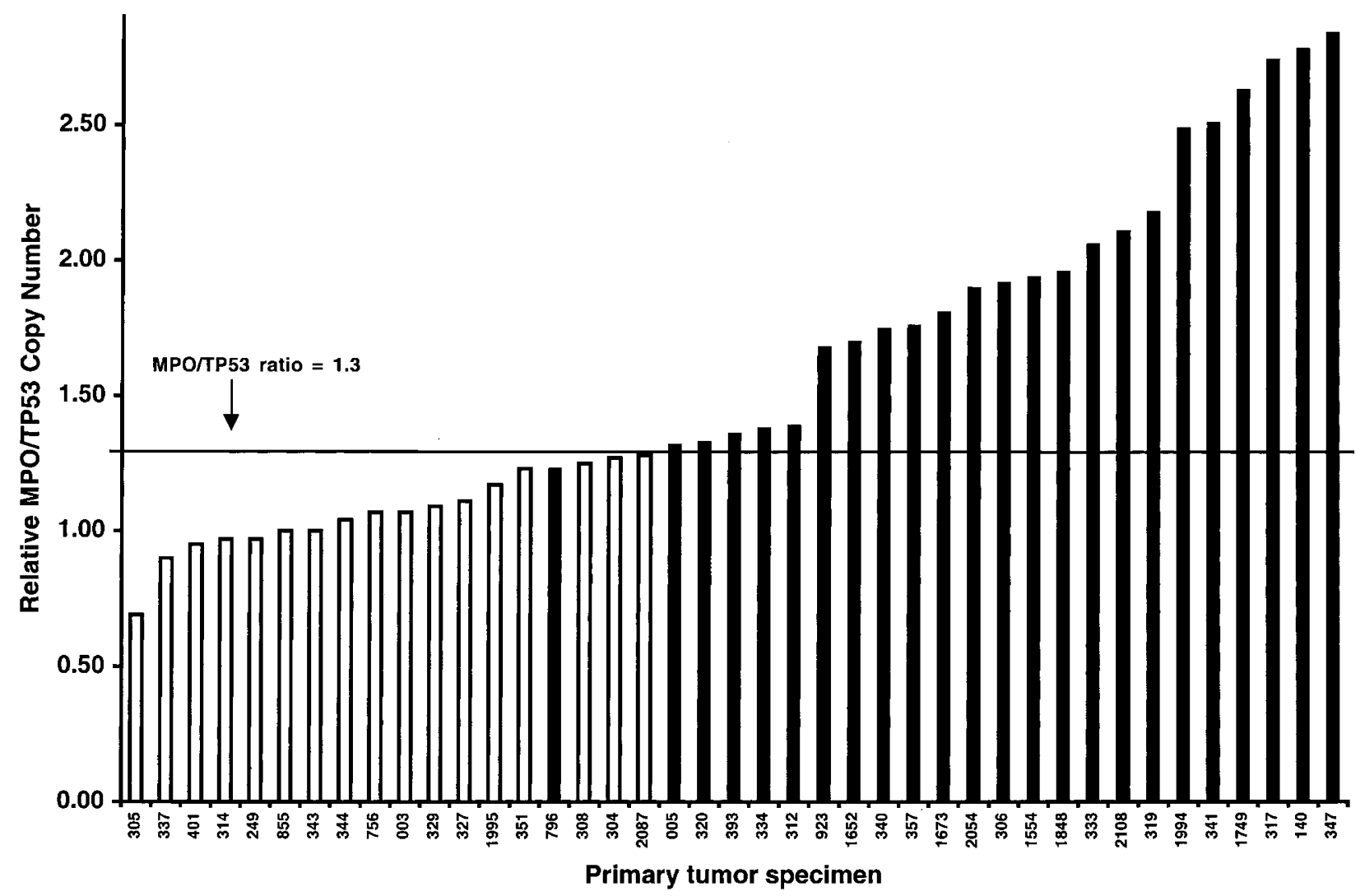

FIGURE 4. Chromosome 17 status of primary human neuroblastoma specimens, as determined by Q-PCR. Tumor samples with $17 \mathrm{q}$ gain as determined by FISH are represented as open bars, whereas those with 17q gain determined by FISH are seen as filled bars. All tumor samples with unbalanced 17q gain by FISH showed relative 17q copy number ratios by Q-PCR of $>1.3$, with the exception of sample 796 .

utility of such information, as MYCN oncogene copy number status is used worldwide for risk stratification. Ongoing research is aimed at refining our current risk stratification algorithm by introducing additional tumor-specific prognostic markers that improve our ability to predict tumor behavior.

The current literature supports a strong association between unbalanced gain of chromosome $17 \mathrm{q}$ material and an adverse clinical outcome $(8,9,18$, 22-24). In addition, 17q copy number status may be an independent predictor of outcome even when patient age at diagnosis, disease stage, and MYCN amplification status are considered (9). The major obstacle for incorporating this marker into routine molecular diagnostics is the technical difficulty that is associated with detecting relatively subtle copy number alterations by FISH. This technique is labor intensive, costly, and of low throughput. Therefore, a reliable PCR-based assay that can be used for the high-throughput screening of $17 \mathrm{q}$ aberrations in diagnostic neuroblastoma samples would allow for definitive clinical correlative studies aimed at determining the clinical utility of this potential prognostic marker.

Real-time quantitative PCR has been adapted to detect high-level genomic amplification in solid tumor samples, including neuroblastoma (25). How- ever, quantitative assessment of relatively subtle copy number gain in clinical samples has not been reported. The assay described here was designed to provide a relative $17 q-17 p$ copy number ratio because unbalanced gain of $17 \mathrm{q}$ material has been shown to be prognostically relevant $(9,10,14,26$, 27). Primer and probe sets were designed based on previous mapping and clinical correlative studies using FISH probes to detect relative abundance of MPO (17q23.1) compared with TP53 (17p13.1) (9, 11). The assay was optimized to allow for use of the comparative Ct method for determining gene copy number, allowing for high throughput by obviating the need for an experiment-specific serial dilution

TABLE 4. Comparison of Q-PCR Data with FISH Analysis for Relative 17q Copy Number Using Different Q-PCR Cut Points

\begin{tabular}{rrr}
\hline & \multicolumn{2}{c}{ FISH } \\
\cline { 2 - 3 } & Gain & No Gain \\
\hline Q-PCR & & \\
$\geq 1.2$ & 23 & 4 \\
$<1.2$ & 0 & 13 \\
$\geq 1.3$ & 22 & 0 \\
$<1.3$ & 1 & 17 \\
$\geq 1.4$ & 17 & 0 \\
$<1.4$ & 6 & 17 \\
\hline
\end{tabular}


to allow for construction of a standard curve. Data from established cell lines showed the technique to correlate well with interphase FISH data, thus validating the experimental strategy in the artificial setting of uncontaminated cellular material.

For this assay to be of clinical utility, it must be both a sensitive and specific method for detecting unbalanced $17 \mathrm{q}$ gain in clinical samples. Neuroblastomas show varying degrees of "contaminating" normal tissues, including vascular and supporting stroma and infiltrating Schwann cells (28). Our data suggest that despite intratumoral cellular heterogeneity, Q-PCR can unequivocally determine $17 q$ allelic status in the majority of clinical samples. In the relatively small sample set studied here, a relative $17 \mathrm{q}$ copy number value of 1.3 provided optimal discrimination between tumors with and without unbalanced $17 \mathrm{q}$ gain. However, it is also apparent that Q-PCR will provide continuous data across a large sample set, suggesting that this PCRbased technique will not be capable of providing $100 \%$ sensitivity and specificity. This should not be surprising, as a significant subset of samples often cannot be reliably classified for $17 \mathrm{q}$ status using FISH. Therefore, we plan to score samples with relative $17 q$ copy number values of 1.2-1.4 in future studies as indeterminate by Q-PCR. Each of these samples will then also be examined by dual-color interphase FISH in an attempt to unequivocally assign $17 \mathrm{q}$ allelic status for all specimens. Ongoing studies in a much larger number of samples may determine a more precise relative $17 q$ copy number cutoff value and range for samples requiring FISH evaluation.

Q-PCR as described here provides for high throughput screening of relative $17 \mathrm{q}$ copy number in primary neuroblastoma specimens. Further studies may refine this assay toward near-perfect sensitivity and specificity, but we expect that there will always be a subset of tumors that should also be studied by FISH if unequivocal determination of $17 \mathrm{q}$ allelic status is the goal. Even without further refinement, this assay will allow for highly specific $17 q$ status assignment for the vast majority of neuroblastomas, thus streamlining integration of this molecular marker into clinical correlative studies. Results from prospective evaluation of the clinical significance of $17 \mathrm{q}$ aberrations in large numbers of human neuroblastomas may lead to refinements in risk stratification algorithms and thus to more precise allocation to therapeutic protocols.

Acknowledgments: This work was supported in part by and NIH Grants R01-CA87847 (J.M.M.) and U01CA30969 (Children's Oncology Group), and a Hope Street Kids Cancer Research Foundation Grant (Q.W. and J.M.M). The authors would like to thank Ms.
Luanne Wainwright for technical assistance and Dr. Hiro Shimada for providing the central review of neuroblastoma histopathology.

\section{REFERENCES}

1. Brodeur GM, Maris JM. Neuroblastoma. In: Pizzo PA, Poplack DG, editors. Principles and practice of pediatric oncology. 4th ed. Philadelphia: Lippincott; 2002. p. 895-938.

2. Maris JM, Matthay KK. Molecular biology of neuroblastoma. J Clin Oncol 1999;17:2264-79.

3. Brodeur G, Seeger RC, Schwab M, Varmus HE, Bishop JM. Amplification of $\mathrm{N}$ - myc in untreated human neuroblastomas correlates with advanced disease stage. Science 1984;224: 1121-4.

4. Seeger RC, Brodeur GM, Sather H, Dalton A, Siegel SE, Wong $\mathrm{KY}$, et al. Association of multiple copies of the N-myc oncogene with rapid progression of neuroblastomas. N Engl J Med 1985;313:1111-6.

5. Shimada H, Ambros IM, Dehner LP, Hata J, Joshi VV, Roald B. The International Neuroblastoma Pathology Classification (Shimada) System. Cancer 1999;86:364-72.

6. Look AT, Hayes FA, Shuster JJ, Douglass EC, Castleberry RP, Bowman LC, et al. Clinical relevance of tumor cell ploidy and $\mathrm{N}-m y c$ gene amplification in childhood neuroblastoma: a pediatric oncology group study. J Clin Oncol 1991;9:581-91.

7. Van Roy N, Laureys G, Cheng NC, Willem P, Opdenakker G, Versteeg R, et al. 1;17 translocations and other chromosome 17 rearrangements in human primary neuroblastoma tumors and cell lines. Genes Chromosomes Cancer 1994;10:103-14.

8. Caron H. Allelic loss of chromosome 1 and additional chromosome 17 material are both unfavourable prognostic markers in neuroblastoma. Med Pediatr Oncol 1995;24:215-21.

9. Bown N, Cotterill S, Lastowska M, O'Neill S, Pearson AD, Plantaz D, et al. Gain of chromosome arm 17q and adverse outcome in patients with neuroblastoma. N Engl J Med 1999;340:1954-61.

10. Lastowska M, Roberts $P$, Pearson AD, Lewis I, Wolstenholme J, Bown N. Promiscuous translocations of chromosome arm $17 \mathrm{q}$ in human neuroblastomas. Genes Chromosomes Cancer 1997;19:143-9.

11. Meddeb M, Danglot G, Chudoba I, Venuat AM, Benard J, Avet-Loiseau H, et al. Additional copies of a $25 \mathrm{Mb}$ chromosomal region originating from 17q23.1-17qter are present in $90 \%$ of high-grade neuroblastomas. Genes Chromosomes Cancer 1996;17:156-65.

12. Savelyeva L, Corvi R, Schwab M. Translocation involving $1 p$ and $17 \mathrm{q}$ is a recurrent genetic alteration of human neuroblastoma cells. Am J Hum Genet 1994;55:334-40.

13. McConville CM, Dyer S, Rees SA, Luttikhuis ME, McMullan DJ, Vickers SJ, et al. Molecular cytogenetic characterization of two non-MYCN amplified neuroblastoma cell lines with complex t(11;17). Cancer Genet Cytogenet 2001;130:133-40.

14. Stark B, Jeison M, Bar-Am I, Glaser-Gabay L, Mardoukh J, Luria D, et al. Distinct cytogenetic pathways of advancedstage neuroblastoma tumors, detected by spectral karyotyping. Genes Chromosomes Cancer 2002;34:313-24.

15. Brodeur GM, Pritchard J, Berthold F, Carlsen NLT, Castel V, Castleberry RP, et al. Revisions in the international criteria for neuroblastoma diagnosis, staging, and response to treatment. J Clin Oncol 1993;11:1466-77.

16. Mathew P, Valentine MB, Bowman LC, Rowe ST, Nash MB, Valentine VA, et al. Detection of MYCN gene amplification in neuroblastoma by fluorescence in situ hybridization: a pediatric oncology group study. Neoplasia 2001;3:105-9.

17. Plantaz D, Mohapatra G, Matthay KK, Pellarin M, Seeger RC, Feuerstein BG. Gain of chromosome 17 is the most frequent 
abnormality detected in neuroblastoma by comparative genomic hybridization. Am J Pathol 1997;150:81-9.

18. Brinkschmidt C, Christiansen $H$, Terpe HJ, Simon R, Lampert F, Boecker W, et al. Distal chromosome 17 gains in neuroblastomas detected by comparative genomic hybridization (CGH) are associated with a poor clinical outcome. Med Pediatr Oncol 2001;36:11-3.

19. Lastowska M, Cotterill S, Bown N, Cullinane C, Variend S, Lunec J, et al. Breakpoint position on 17q identifies the most aggressive neuroblastoma tumors. Genes Chromosomes Cancer 2002;34:428-36.

20. Trakhtenbrot L, Cohen N, Betts DR, Niggli FK, Amariglio N, Brok-Simoni F, et al. Interphase fluorescence in situ hybridization detection of chromosome 17 and $17 q$ region gains in neuroblastoma: are they secondary events? Cancer Genet Cytogenet 2002;137:95-101.

21. Applied Biosystems. ABI PRISM 7700 Sequence Detection System User Guide. In: User Bulletin \#2: Applied Biosystems.

22. Abel F, Ejeskar K, Kogner P, Martinsson T. Gain of chromosome arm $17 \mathrm{q}$ is associated with unfavourable prognosis in neuroblastoma, but does not involve mutations in the somatostatin receptor 2(SSTR2) gene at 17q24. Br J Cancer 1999;81:1402-9.

23. Lastowska M, Cotterill S, Pearson AD, Roberts P, McGuckin A, Lewis I, et al. Gain of chromosome arm $17 \mathrm{q}$ predicts unfavourable outcome in neuroblastoma patients. U.K. Children's Cancer Study Group and the U.K. Cancer Cytogenetics Group. Eur J Cancer 1997;33:1627-33.

24. Lastowska M, Cullinane C, Variend S, Cotterill S, Bown N, O'Neill S, et al. Comprehensive genetic and histopathologic study reveals three types of neuroblastoma tumors. J Clin Oncol 2001;19:3080-90.

25. De Preter K, Speleman F, Combaret V, Lunec J, Laureys G, Eussen BH, et al. Quantification of MYCN, DDX1, and NAG gene copy number in neuroblastoma using a real-time quantitative PCR assay. Mod Pathol 2002;15: 159-66.

26. Cunsolo CL, Bicocchi MP, Petti AR, Tonini GP. Numerical and structural aberrations in advanced neuroblastoma tumours by CGH analysis; survival correlates with chromosome 17 status. Br J Cancer 2000;83:1295-300.

27. Lastowska M, Van Roy N, Bown N, Speleman F, Lunec J, Strachan T, et al. Molecular cytogenetic delineation of $17 \mathrm{q}$ translocation breakpoints in neuroblastoma cell lines. Genes Chromosomes Cancer 1998;23:116-22.

28. Ambros IM, Zellner A, Roald B, Amann G, Ladenstein R, Printz D, et al. Role of ploidy, chromosome 1p, and Schwann cells in the maturation of neuroblastoma. N Engl J Med 1996;334:1505-11. 